CD4 $+\mathrm{T}$ cells and regulatory T cells on day 20 in $\alpha$-IL-10R MSEW mice compared to NR counterparts. This difference disappeared by day 30 . Histological scoring showed no difference in disease severity between $\alpha$-IL-10R treated MSEW and NR mice on day 20. However, on day 30, when $\alpha$-IL-10R NR mice are recovering from colitis, MSEW mice showed persistent histological inflammation, mainly attributable to sustained epithelial damage. DISCUSSION/ SIGNIFICANCE OF IMPACT: Our results suggest that ELS prolongs intestinal inflammation and impairs epithelial repair. Future studies will focus on elucidating the mechanisms responsible for ELS-dependent impairment of mucosal repair in experimental colitis.

\section{Evaluating the effect of a compliant stent-graft prototype on effective stiffness in a cadaveric aorta} Shannen B Kizilski ${ }^{1}$, Filippo Coletti, $\mathrm{PhD}^{2}$, Rumi Faizer, $\mathrm{MD}^{2}$, and Victor H. Barocas, $\mathrm{PhD}^{2}$

${ }^{1}$ University of Minnesota CTSI; ${ }^{2}$ University of Minnesota

OBJECTIVES/GOALS: High aortic stiffness is associated with increased cardiovascular morbidity and mortality. The purpose of this work is to demonstrate the potential of our compliant stent-graft design to therapeutically increase aortic compliance over a standard aortic stent-graft. METHODS/STUDY POPULATION: The aorta from a human cadaver will be excised and placed into a pulse duplicator circuit. The stiffness of the system will be estimated using the pulse wave velocity (PWV), which will be calculated using the time delay between pressure measurements at proximal and distal locations in the system. Baseline measurements with the unstented aorta will be compared to two cases: (1) with a standard stent-graft placed, and (2) with our compliant stent-graft prototype in the descending thoracic aorta. PWV is calculated as the distance between the pressure sensors divided by the time delay. Faster PWV is associated with a stiffer vessel, or lower aortic compliance. RESULTS/ANTICIPATED RESULTS: Prior work in vitro showed that the compliant stent-graft reduced peak and pulse pressures compared a standard, rigid stent-graft. We also expect the compliant device to exhibit lower PWV compared to a rigid stent-graft. Depending on the aortic tissue stiffness, the compliant stent-graft could raise or lower PWV compared to no stent. Mean pressure in the compliant case is likely to be slightly higher than the other two cases because the compliant stent-graft's narrower lumen increases flow resistance. Although mean pressure will be higher, peak pressure should be lower than in the standard stent-graft because the added compliance decreases overall pressure swing between systole and diastole. DISCUSSION/SIGNIFICANCE OF IMPACT: Lower PWV in the compliant stent-graft over the standard stent-graft will indicate its potential to therapeutically lower aortic stiffness in patients needing aortic stenting. Positive outcomes from this study will be a step toward the eventual translation of a compliant stent-graft to clinical use.

4287

Extracellular vesicles as biomarkers for early detection of pancreatic cancer

Charles P Hinzman ${ }^{1}$, Shivani Bansal ${ }^{2}$, Yaoxiang $\mathrm{Li}^{2}$, Partha

Banerjee $^{2}$, and Amrita Cheema ${ }^{2}$

${ }^{1}$ Georgetown - Howard Universities; ${ }^{2}$ Georgetown University Medical Center

OBJECTIVES/GOALS: Pancreatic ductal adenocarcinoma (PDAC) is projected to become the second leading cause of cancer-related deaths by 2030. Though many other cancers have seen improvements in patient survival rates, patients diagnosed with PDAC have a 5-year survival rate of only $~ 9 \%$. A major contributor to decreased survival is late-stage diagnosis of the disease. New methods of early detection are urgently needed. Extracellular vesicles (EVs) are secreted from cells of all tissue types into the circulation. EVs play important roles in a variety of diseases. They have shown to promote cancer progression and they are being studied as potential biomarkers for disease diagnosis. The purpose of this study was to perform qualitative and quantitative characterization of smallmolecule profiles of EVs derived from various pancreatic cancer (PC) and normal pancreas cell lines, to provide proof-of-concept for evaluating the efficacy of leveraging EVs as potential biomarkers of PDAC. METHODS/STUDY POPULATION: EVs were isolated from the conditioned media of six PC and two normal pancreas cell lines using differential ultracentrifugation with filtration. EV enrichment was validated using quantitative ELISA, immunoblot and transmission electron microscopy. Targeted liquid chromatography coupled to mass spectrometry (LC-MS/MS) and untargeted (UPLC-QTOF-MS) metabolomics were used to analyze the biochemical composition of EVs. RESULTS/ANTICIPATED RESULTS: The biochemical profile of PC EVs was found to be significantly different from the profiles of normal cell EVs. Interestingly, amino acids were downregulated in PC EVs as compared to normal cell EVs. However, PC EVs were enriched in lactate and malate. PC EVs also had significant upregulation in other small molecules such as xanthosine, guanosine diphosphate and nicotinamide. DISCUSSION/SIGNIFICANCE OF IMPACT: Our results indicate that the biochemical characterization of EVs using metabolomics has the potential to yield biomarkers which can delineate cancer cell-derived EVs from normal cell-derived EVs. Further work will test the clinical significance of these findings by similar analyses of plasma of PDAC patients. Furthermore, these profiles may be detectable before progression of the disease to late-stage PDAC, leading to the development of assays for earlier diagnosis in patients.

4543

Glucocorticoid Receptors are essential effectors of TGF $\beta$ signaling in Triple Negative Breast Cancer

Carlos Jesus Perez Kerkvliet ${ }^{1}$, Amy R Dwyer, Caroline Diep, Robert Oakley, Christopher Liddle, and Carol A Lange

${ }^{1}$ University of Minnesota CTSI

OBJECTIVES/GOALS: The glucocorticoid receptor (GR) is a ubiquitous steroid hormone receptor that is emerging as a mediator of breast cancer metastasis. We aim to better understand the biology associated with phospho-GR species in TNBC and their contribution to tumor progression. METHODS/STUDY POPULATION: To better understand how p-S134 GR may impact TNBC cell biology, we probed GR regulation by soluble factors that are rich within the tumor microenvironment (TME), such as TGF $\beta$. TNBC cells harboring endogenous wild-type or S134A-GR species were created by CRISPR/Cas knock-in and subjected to in vitro assays of advanced cancer behavior. RNA-Seq was employed to identify pS134-GR target genes that are uniquely regulated by TGF $\beta$ in the absence of exogenously added GR ligands. Direct regulation of selected TGF $\beta$-induced $\mathrm{pS} 134$-GR target genes was validated accordingly. Bioinformatics tools were used to probe publicly available TNBC patient data sets for expression of a pS134-GR 24-gene signature. RESULTS/ANTICIPATED RESULTS: In the 
absence of GR ligands, GR is transcriptionally activated via p38MAPK-dependent phosphorylation of Ser134 upon exposure of TNBC cells to TME-derived agents (TGF $\beta$, HGF). The ligandindependent pS134-GR transcriptome primarily encompasses gene sets associated with TNBC cell survival and migration/invasion. Accordingly, pS134-GR was essential for TGF $\beta$-induced TNBC cell migration, anchorage-independent growth in soft-agar, and tumorsphere formation, an in vitro readout of breast cancer stemness properties. Finally, a 24-gene pSer134-GR-dependent signature induced by TGF $\beta 1$ predicts shortened survival in breast cancer. We expect to find similar results using an in-house tissue microarray. DISCUSSION/SIGNIFICANCE OF IMPACT: Phospho-S134-GR is a critical downstream mediator of p38 MAPK signaling and TNBC migration, survival, and stemness properties. Our studies define GR as a required effector of TGF $\beta 1$ signaling and nominate $\mathrm{pS} 134-\mathrm{GR}$ as a biomarker of elevated risk of breast cancer dissemination.

\section{Identification of small molecules that facilitate the efficient differentiation of stem cell derived $\beta$-cells ${ }^{*}$ Yuhao Min ${ }^{1}$, Chris Clifford, and Quinn P. Peterson \\ ${ }^{1}$ Mayo Clinic}

OBJECTIVES/GOALS: In this study, we established a highthroughput chemical screening platform to identify small molecules that facilitates efficient differentiation of stem cells derived $\beta$ (SC- $\beta$ ) cells. Using this platform, we identified several compounds that potentially increase the differentiation efficiency. METHODS/ STUDY POPULATION: Differentiation of human embryonic stem cells (HUES8) into SC- $\beta$ was carried out using previously published protocols in a $3 \mathrm{D}$ cell suspension. Single cells were replated in Matrigel-coated well plates at the start of different stages depending on experiments. Differentiation medium supplemented with small molecules at a final concentration of $2 \mathrm{M}$ and $0.2 \mathrm{M}$ was used throughout the stage. All the cells were then fixed and permeabilized. Immunocytochemical staining was performed. Images of each well were taken and analyzed. Numbers of the total cell, insulinpositive cell, NKX6.1-positive cell, and co-positive cell were recorded. Candidate compounds were validated using flow cytometry or ICC. RESULTS/ANTICIPATED RESULTS: We identified several hit compounds that significantly increase the NKX6.1 positive cell percentage compared to the DMSO-treated controls when treated at the PP1 cell stage. Follow up assays demonstrated that at least one of these putative hits reproducibly increased NKX6.1 expression. In addition, we identified other compounds that significantly increase the insulin and NKX6.1 copositive SC- $\beta$ cell population when treated at the later PP2 cell stage during the differentiation. We expect a dosage-dependent response when the candidate hits are validated using more accurate assays. DISCUSSION/SIGNIFICANCE OF IMPACT: We established a high-throughput screening platform to identify small molecules that increase the efficiency of SC- $\beta$ direct differentiation. Successful generation of SC- $\beta$ allows cell replacement therapy in diabetes patients, and a better understanding of pancreatic biology and development.
Impaired Natural Killer Cell Function May Be Associated with Cancer-related Fatigue

Jeniece Regan ${ }^{1}$, Rebekah Feng, and Leorey Saligan

${ }^{1}$ National Institutes of Health

OBJECTIVES/GOALS: During and after cancer treatment, cancerrelated fatigue $(\mathrm{CRF})$ is a debilitating symptom reported by up to $80 \%$ of cancer patients Our understanding of the pathology underlying $\mathrm{CRF}$ is limited. Preliminary RNA sequencing data suggest that increased levels of KIR3DL1, the natural killer cell (NK) immunoglobulin-like receptor 3DLla, may be associated with CRF. METHODS/STUDY POPULATION: Fatigue was measured using the Functional Assessment of Chronic Illness Therapy-Fatigue (FACT-F). Functional validation of the NK cell finding was performed from whole blood obtained from fatigued and non-fatigued subjects. NK cells were isolated from freshly collected whole blood using a human NK cell isolation kit based on CD56 microbead positive selection. NK cell function was assessed using the NK cell direct cytotoxicity assay. Briefly, isolated NK cells were co-cultured in a 2:1 ratio with calcein AM-labelled K562 cells, which are NK cell-sensitive due to the very low MHCI expression. NK cell-mediated cytotoxicity was assessed with Cytation 1 Cell Imaging Multi-Mode reader. Flow cytometric protocols were used to examine NK subset differences between the fatigued and non-fatigued groups. RESULTS/ANTICIPATED RESULTS: NK cells isolated from the fatigued group exhibited decreased cytotoxicity at $12.28 \%$ compared to NK cells isolated from non-fatigued controls at a mean of $40.6 \%$ cytotoxicity. Flow cytometry analysis revealed a decrease in the $\mathrm{CD} 56^{\mathrm{dim}} \mathrm{CD} 16^{\text {bright }}$ population in the fatigued group (87.1\% of CD56+CD4- cells) compared to the control $(91.4 \%$ of CD56+CD4- cells). Furthermore, there was a decrease in NKG2A expression in mature $\mathrm{NK}$ cells $\left(\mathrm{CD} 56^{\mathrm{dim}} \mathrm{CD} 16^{\text {bright }}\right.$ ) isolated from the fatigued group compared to the non-fatigued group. DISCUSSION/SIGNIFICANCE OF IMPACT: Results from the pilot study suggest that there was a decrease in NK cell cytotoxicity in the fatigued group. In addition, there may be a shift in NK cell subpopulations associated with fatigue. Findings from this pilot study suggest that impaired NK cell function may be associated with CRF pathogenesis.

4323

\section{Inflammation partially mediates fatigue-like behavior in mice}

Sarah Alshawi ${ }^{1}$, Brian Wolff ${ }^{1}$, and Leorey Saligan ${ }^{1}$

${ }^{1}$ National Institutes of Health

OBJECTIVES/GOALS: Fatigue is a distressing side effect of cancer and its treatment. It is a subjective symptom that can include mental, physical, emotional, and motivational components. We sought to determine whether preventing inflammation affects fatigue-like behavior in a mouse model of radiation therapy. METHODS/ STUDY POPULATION: C57BL/6 mice received three consecutive 8 gray doses of daily peripheral irradiation. We used voluntary wheel running activity to measure fatigue-like behavior before and after this period. Minocycline, an antibiotic with anti-inflammatory effects, was administered beginning a week before irradiation and 